\title{
O cenário da assistência de enfermagem frente aos pacientes surdos: revisão integrativa
}

\author{
The nursing assistance scenario in front of deaf patients: integrative review
}

El escenario de la atención de enfermería al paciente sordo: una revisión integradora

\author{
Kamila Araujo Vieira1*, Fabrícia Corrêa de Brito ${ }^{1}$, Marcos Vinicius Costa Fernandes ${ }^{1}$.
}

\begin{abstract}
RESUMO
Objetivo: Descrever que uma comunicação efetiva entre o profissional de saúde e o paciente surdo em uma unidade de saúde é importante e se torna fundamental para a garantia da integridade do paciente surdo. Métodos: A pesquisa teve como objetivo identificar as dificuldades de assistência aos indivíduos com surdez e avaliar a concepção dos profissionais de saúde no processo de cuidar. É uma revisão integrativa da literatura científica de artigos publicados no período de 5 anos (2015 a 2020) na BDENF, LILACS e SciELO. Resultados: Após o estudo, 10 artigos identificaram a questão: "Qual a qualidade da assistência prestada aos pacientes com surdez nas unidades de saúde? " Como uma das normas da pesquisa, que foi abordada na discussão: 1. Déficit na assistência prestada ao paciente surdo. 2. Falta de conhecimento sobre Libras. 3. Falta de recursos adequados. Considerações finais: Foi descoberto que existem barreiras no serviço, indicando falta de experiência da equipe e que o despreparo dos profissionais afeta a qualidade de vida do paciente.
\end{abstract}

Palavras-chave: Surdez, Comunicação, Saúde.

\begin{abstract}
Objective: To describe that an effective communication between the health professional and the deaf patient in a health unit is important and becomes essential to guarantee the integrity of the deaf patient. Methods: The research aimed to identify the difficulties in assisting individuals with deafness and to evaluate the conception of health professionals in the care process. It is an integrative review of the scientific literature of articles published in the period of 5 years (2015 to 2020) in BDENF, LILACS and SciELO. Results: After analysis, 10 articles found, among the research expectations, the question: "What is the quality of care provided to patients with deafness in health units?", as one of the research norms, which was addressed in the discussion: 1. Deficit in care provided to deaf patients. 2. Lack of knowledge about Libras. 3. Lack of adequate resources. Final considerations: It was discovered that there are barriers in the service, indicating a lack of staff experience and that the practitioners' unpreparedness affects the patient's quality of life.
\end{abstract}

Keywords: Deafness, Communication, Health.

\section{RESUMEN}

Objetivo: Describir que una comunicación efectiva entre el profesional de la salud y el pacientes ordo en una unidad de salud es importante y se vuelve fundamental para garantizar la integridad del paciente sordo. Métodos: La investigación tuvo como objetivo identificar las dificultades para asistir a las personas con sordera y evaluar la concepción de los profesionales de la salud en el proceso de atención. Es una revisión integradora de la literatura científica de los artículos publicados en el período de 5 años (2015 a 2020) en BDENF, LILACS y SciELO. Resultados: Después del estudio, 10 artículos identificaron la pregunta: “¿Cuál es la calidad de la atención a los pacientes sordos en las unidades de salud?" como una de las normas de investigación, que se abordó en la discusión: 1. Déficit en la asistencia brindada al paciente sordo. 2. Falta de conocimiento sobre Libra. 3. Falta de recursos adecuados. Consideraciones finales: Se descubrió que existen barreras en el servicio, lo que indica la falta de experiencia del equipo y que la falta de preparación del profesional afecta la calidad de vida del paciente.

Palabras clave: Sordera, Comunicación, Salud.

${ }^{1}$ Faculdade Estácio do Amazonas, Manaus - AM. *E-mail: kamilaav5@gmail.com 


\section{INTRODUÇÃO}

A comunicação constitui-se como uma das bases de estrutura da sociedade, pois engloba todas as áreas de convívio dos seres humanos, desde o processamento da convivência social, como cultural, político e econômica. Além de compor os fundamentos das lutas de sobrevivência da humanidade no decorrer dos anos, baseando-se na procura de conhecimentos acerca do mundo. Diante disso, podemos conceituar à conversação como um procedimento primordial em todos os tipos de relações. Contudo, uma comunicabilidade só pode ser considerada adequada quando a mensagem é obtida com o mesmo intuito na qual foi enviada, sendo capaz de ser realizada de diversas maneiras, mediante da linguagem verbal ou não verbal, desde que seja efetuada de forma coerente e completa (OLIVEIRA YCA, et al., 2015).

Com relação à população com surdez, inúmeras vezes existem empecilhos nesta comunicação, a qual pode prejudicar a interação social destes indivíduos ou até mesmo a inter-relação do usuário com o profissional, levando a respeito que a ausência da conversação por intermédio da fala torna o surdo desintegrado da sociedade ouvinte. Apresentando assim, um obstáculo neste entrosamento, pois os ouvintes possuem dificuldades no entendimento da língua de sinais, o que gera para o surdo uma problemática em usufruir os serviços básicos essenciais da vida, tendo como exemplo, o seu acesso aos hospitais (OLIVEIRA YCA, et al., 2015).

Desde os primórdios da humanidade o cidadão surdo enfrenta embates em relação a sua condição. Haja vista, que antigamente as pessoas com deficiência eram consideradas ora amaldiçoadas, ora seres semidivinos, mas, sempre eram desprezadas do contexto social e vistas como ferramenta de caridade da comunidade. Nas civilizações gregas de Esparta e Atenas, os nascidos com qualquer tipo de deficiência eram eliminados, uma vez que, os espartanos veneravam o corpo físico e começavam a preparar os meninos desde os setes anos de idade para serem capacitados durante as guerras e defesa da polis, enquanto, os atenienses cultuavam o intelecto e seus esforços eram para a realização das atividades artísticas, culturais e filosóficas. Dessa forma, essa população era considerada inválida e não conseguiram adequar-se ao Exército de Esparta e nem obter as instruções em Atenas (DUARTE SBR, et al., 2013).

No século VI, foi relatado pela primeira vez uma das mais antigas classificações para os surdos, por entremeio do direito e da saúde, através do Código Justiniano, que os classificavam em cinco categorias: surdo-mudez natural; surdo-mudez adquirida; surdez natural; surdez adquirida; mudez natural ou adquirida. Essa rotulação mudou as condições socioantropológicas e estabeleceu a diferença de tratamento dos surdos falantes e o que não falavam. Os que não conseguiam se comunicar pela fala eram impossibilitados mediante a lei de firmar contratos, adquirir propriedades, reivindicar herança, desenvolver testamentos e principalmente de viver em comunidade. Essas normas e diretrizes não enquadraram os cidadãos surdos que falavam, dado que, a maior parte eram aqueles que possuíram em primeiro lugar a linguagem e posteriormente a condição da surdez (DUARTE SBR, et al., 2013).

As definições de surdez sofreram muitas alterações ao longo dos séculos, seguindo os métodos técnicos evolutivos da ciência, atualmente diante o Decreto 5.626, de 22 de dezembro de 2005, no Art. 20 "considerase pessoa surda aquela que, por ter perda auditiva, compreende e interage com o mundo por meio de experiências visuais, manifestando sua cultura principalmente pelo uso da Língua Brasileira de Sinais Libras". Ainda a respeito dessa lei, recebe o diagnóstico de deficiente auditivo quando o indivíduo tem perda bilateral, parcial ou total, de quarenta e um decibéis $(\mathrm{dB})$ ou mais, aferida por audiograma nas frequências de $500 \mathrm{~Hz}, 1.000 \mathrm{~Hz}, 2.000 \mathrm{~Hz}$ e $3.000 \mathrm{~Hz}$ (BRASIL, 2005).

Apesar da Lei 10.098/00 dispor à acessibilidade a todos os portadores de qualquer deficiência e sua inserção igualitária na sociedade, e do Art. 196 da Constituição Federal de 1988 que impõe a saúde como um direito de todos e dever do Estado, somente através da Lei 10.436/02 que a comunidade com surdez garantiu oficialmente o reconhecimento legal da comunicação e expressão a Língua Brasileira de Sinais (LIBRAS) e outros recursos de expressão a ela associado, além de sustentar as concessões da inclusão plena dos sujeitos com deficiência auditiva aos atendimentos por profissionais capacitados de acordo com as normas legais em vigor e os princípios do Sistema Único de Saúde (SUS) (OLIVEIRA WSR, et al., 2020; SOUZA MFNS, et al., 2017). 
Diante do Conselho Federal de Enfermagem (COFEN), no capítulo II dos Deveres: Art. 55 "Aprimorar os conhecimentos técnico-científicos, ético-políticos, socioeducativos e culturais, em benefício da pessoa, família e coletividade e do desenvolvimento da profissão". Assegurando também no Art. 41 "Prestar assistência de enfermagem sem discriminação de qualquer natureza". Ademais, é necessário haver uma inserção sociocultural e biopsicossocial de cada indivíduo nas instituições de saúde, formulando maneiras para fortalecer a interação social entre esse grupo e a recepção do profissional (CONSELHO FEDERAL DE ENFERMAGEM, 2017; ANCHIETA EVB, 2020).

$\mathrm{Na}$ área de saúde a relação profissional-cliente, constitui um processo essencial para a prestação de uma assistência de qualidade, pois abrange, além dos conceitos intrínsecos, a escuta qualificada, na maneira de escuta acolhedora, não apenas como um meio de repassar informações para um entendimento conceitual, mas assimilando as subjetividades dos sujeitos. Todavia, em uma sociedade na qual a língua oral é predominante, por conseguinte, as pessoas precisam adaptar-se a ela para que haja uma interação no meio social, ou seja, a população não está preparada para amparar o ser humano com surdez. Nessa perspectiva, o vínculo entre um cliente surdo e o servidor de saúde pode ser permeado por adversidades na comunicabilidade (OLIVEIRA YCA, et al., 2014).

Consequentemente, ainda são encontradas barreiras de comunicação entre essa população e os profissionais de saúde, mostrando o despreparo dos trabalhadores com os pacientes surdos. Visto que, a LIBRAS poderia ser usada como uma forma de inclusão social e de humanização na assistência prestada pela equipe de saúde, contudo, alguns profissionais encontram-se em uma posição de impotência perante a prestação de serviços e promoção de saúde ao surdo no país, relatando inseguranças e até incapacidade de realizar tal atendimento (TRECOSSI MO e ORTIGARA EPF, 2013; FRANCISQUETI V, et al., 2017; NASCIMENTO BD, et al., 2020).

Logo, é notável que ainda não existe um investimento suficiente para uma inclusão igualitária a esses clientes com surdez, no que se diz respeito, a integração do surdo, dando suporte nos atendimentos necessários e específicos. Dessa forma, faz-se necessário avaliar a assistência de enfermagem frente aos pacientes com surdez.

\section{MÉTODOS}

Trata-se de uma revisão integrativa da literatura científica, com o intuito de selecionar e sintetizar resultados de artigos empíricos relacionados à assistência de enfermagem com pacientes surdos.

Dessa forma, a questão de investigação que norteou o estudo foi: Qual a qualidade do serviço prestado aos clientes com surdez nas unidades de saúde?

A execução deste estudo seguiu as subsequentes etapas: Identificação do tema, a concepção da pergunta norteadora e definição do objetivo, estipulação dos critérios de inclusão e exclusão, seleção dos estudos, verificação dos resultados e análises. Discussão e apresentação das evidências descobertas. A investigação bibliográfica foi realizada nos meses de setembro e outubro do ano de 2020, através das bases de dados Scientific Electronic Library Online (SCIELO), Medical Literature Analysis Americana e do Caribe em Ciências da Saúde (LILACS) e Base de Dados de Enfermagem (BDENF).

Para a busca dos periódicos foram usados os descritores elegidos mediante a consulta dos Descritores em Ciência da Saúde (DeCs), nas respectivas combinações "surdez" AND "comunicação" AND "saúde". Com base na associação desses descritores foram achados 100 artigos. Levando em conta a escolha dos estudos, o recorte temporal estimado foi de 5 anos, ou seja, 2015 a 2020.

Por conseguinte, foi realizada a leitura dos títulos e resumos das 100 publicações com a proposta de lapidar as amostras, por intermédio dos critérios de inclusão e exclusão ou por serem duplicados. Realizouse a leitura completa de 31 artigos e de forma secundária, desenvolveu-se a aplicação do processo de análise temática de conteúdo por artifício da leitura e releitura dos resultados das pesquisas, explorando reconhecer informações importantes que reforçam ou se destacavam. 
Foram incluídos os estudos que haviam sido publicados nos periódicos SCIELO, LILACS e BDENF, que estavam disponíveis gratuitamente, os que possuíssem texto completo e em português e aqueles que corresponderam à publicação no período estabelecido pelo recorte temporal para essa pesquisa. Em relação aos critérios de exclusão foi estipulado: Os títulos que fugiram do tema proposto, artigos duplicados, os em idiomas que não fossem o português brasileiro, além daqueles que não se enquadraram no recorte temporal determinado mediante a análise para esse estudo, a amostragem final foi composta por 10 artigos.

Posteriormente, os dados obtidos foram organizados de maneira concisa, constituindo um banco de dados. As informações estão estruturadas em um quadro contendo: Número do artigo selecionado, base de dados utilizada, periódico de publicação, autor(es), título/ano, e a metodologia aplicada no artigo. O estudo decorreu de uma leitura meticulosa, e em seguida, uma avaliação crítica com foco nas publicações que respondiam aos critérios de inclusão.

\section{RESULTADOS}

Inicialmente foram encontrados 100 artigos, os quais correspondiam às palavras-chaves: surdez, comunicação e saúde. Após a leitura crítica e análise dos critérios de inclusão e exclusão dos trabalhos, 10 periódicos foram selecionados para a pesquisa. Constatou-se que o maior número de estudos foi encontrado na base de dados LILACS, seguido respectivamente pelos periódicos SCIELO e BDENF conforme demonstrado na Figura 1.

Figura 1 - Seleção de artigos para a revisão.
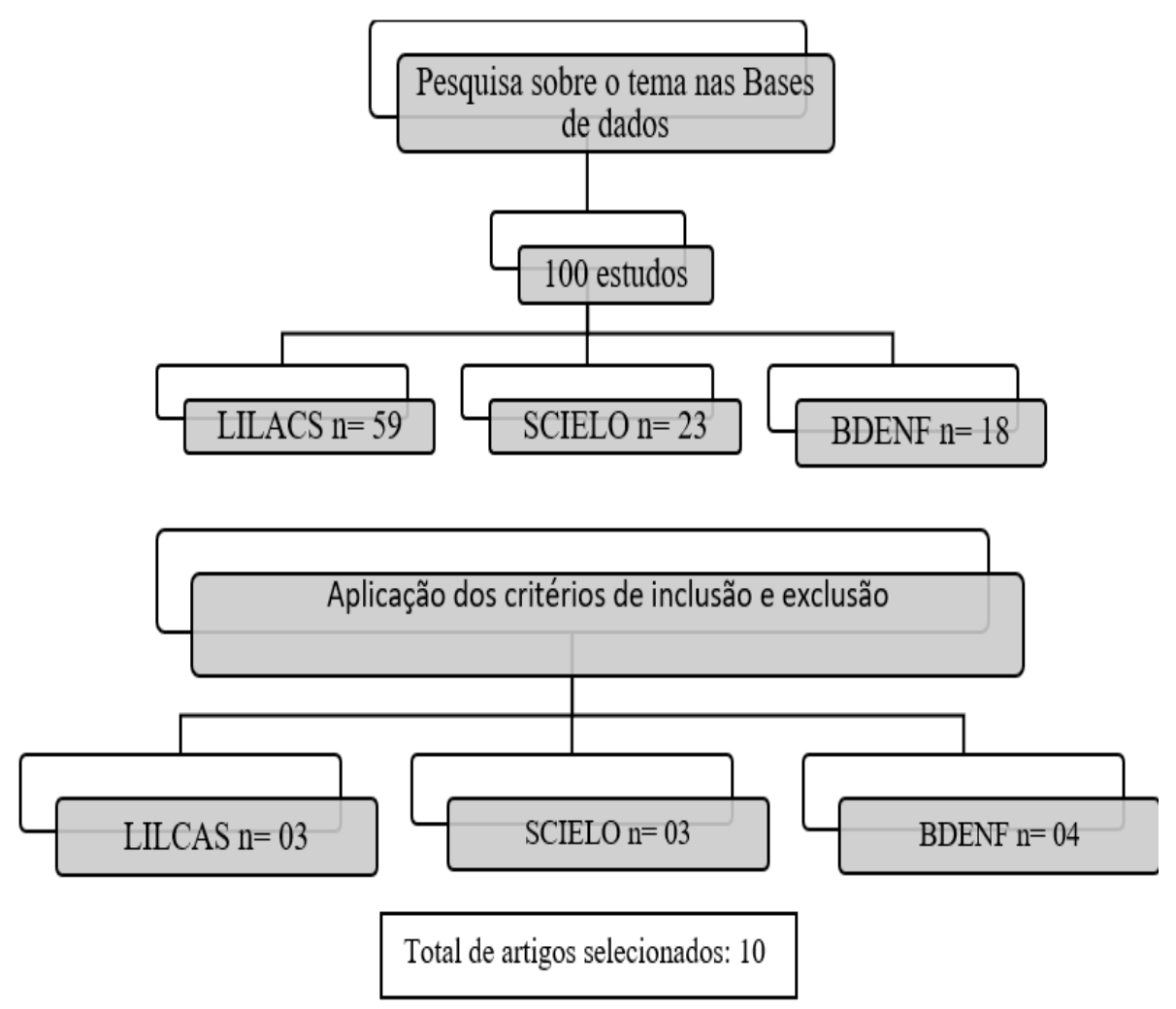

Fonte: Vieira KA, et al., 2021.

Esta revisão é composta por 10 artigos publicados entre 2015 e 2020; destes um artigo publicado respectivamente nos anos 2015, 2016, e 2018. Três foram publicados em 2017, e quatro foram publicados em 2019. Da amostra selecionada três estudos qualitativos, três eram estudos exploratórios descritivos e quatro eram estudos transversais, descritivos e analíticos, conforme demonstrado no Quadro 1. 
Quadro 1 - Artigos e bases de dados utilizados para elaboração da revisão integrativa.

\begin{tabular}{|c|c|c|c|c|c|}
\hline $\mathbf{N}$ & Base & Periódico & Autor(es) & Título/Ano & Metodologia \\
\hline 1 & BDENF & $\begin{array}{l}\text { Rev. Eletrônica } \\
\text { Enferm. }\end{array}$ & $\begin{array}{l}\text { THOMAZ MM, } \\
\text { et al. }\end{array}$ & $\begin{array}{c}\text { Acessibilidade do } \\
\text { adolescente com deficiência } \\
\text { auditiva aos serviços de } \\
\text { saúde/ } 2019 .\end{array}$ & Pesquisa qualitativa. \\
\hline 2 & BDENF & $\begin{array}{l}\text { Rev. Latino - AM } \\
\text { Enfermagem. }\end{array}$ & $\begin{array}{l}\text { SANTOS AS e } \\
\text { PORTES AJF }\end{array}$ & $\begin{array}{c}\text { Percepções de sujeitos } \\
\text { surdos sobre a comunicação } \\
\text { na Atenção Básica à Saúde/ } \\
2019 .\end{array}$ & $\begin{array}{c}\text { Estudo transversal } \\
\text { descritivo e analítico, } \\
\text { com abordagem mista, } \\
\text { qualitativa e } \\
\text { quantitativa. }\end{array}$ \\
\hline 3 & BDENF & Rev. Baiana Enferm. & $\begin{array}{l}\text { SOARES IP, et } \\
\text { al. }\end{array}$ & $\begin{array}{l}\text { Como eu falo com você? A } \\
\text { comunicação do enfermeiro } \\
\text { com o usuário surdo/ } 2018 .\end{array}$ & $\begin{array}{c}\text { Estudo exploratório } \\
\text { descritivo, de } \\
\text { abordagem qualitativa. }\end{array}$ \\
\hline 4 & BDENF & $\begin{array}{c}\text { Revista Enfermagem } \\
\text { Atual. }\end{array}$ & $\begin{array}{l}\text { CAVAGNA VM, } \\
\text { et al. }\end{array}$ & $\begin{array}{l}\text { O paciente surdo e suas } \\
\text { vivências no sistema de } \\
\text { saúde: Uma interface com a } \\
\text { Enfermagem/ } 2017 .\end{array}$ & $\begin{array}{c}\text { Estudo exploratório, } \\
\text { com abordagem } \\
\text { qualitativa. }\end{array}$ \\
\hline 5 & SCIELO & Rev. CEFAC. & $\begin{array}{l}\text { REIS VSL e } \\
\text { SANTOS AM }\end{array}$ & $\begin{array}{c}\text { Conhecimento e experiência } \\
\text { de profissionais das Equipes } \\
\text { de Saúde da Família no } \\
\text { atendimento de pessoas } \\
\text { surdas/ } 2019 .\end{array}$ & Estudo transversal. \\
\hline 6 & SCIELO & $\begin{array}{c}\text { CIENCIA Y } \\
\text { ENFERMERIA XXII. }\end{array}$ & $\begin{array}{l}\text { FRANÇA EG, et } \\
\text { al. }\end{array}$ & $\begin{array}{l}\text { Dificuldades de profissionais } \\
\text { na atenção à saúde da } \\
\text { pessoa com surdez severa/ } \\
2016 \text {. }\end{array}$ & $\begin{array}{l}\text { Estudo descritivo e } \\
\text { transversal. }\end{array}$ \\
\hline 7 & SCIELO & $\begin{array}{l}\text { Interface } \\
\text { Comunicação Saúde } \\
\text { Educação. }\end{array}$ & $\begin{array}{l}\text { OLIVEIRA YCA, } \\
\text { et al. }\end{array}$ & $\begin{array}{l}\text { Conhecimento e fonte de } \\
\text { informações de pessoas } \\
\text { surdas sobre saúde e } \\
\text { doença/ } 2015 .\end{array}$ & $\begin{array}{l}\text { Estudo descritivo- } \\
\text { analítico, com } \\
\text { abordagem qualitativa. }\end{array}$ \\
\hline 8 & LILACS & Saúde em Redes. & $\begin{array}{l}\text { FERREIRA } \\
\text { DRC, et al. }\end{array}$ & $\begin{array}{c}\text { Assistência à gestante } \\
\text { surda: Barreiras de } \\
\text { comunicação encontradas } \\
\text { pela equipe de saúde/ } 2019 \text {. }\end{array}$ & $\begin{array}{l}\text { Estudo descritivo } \\
\text { exploratório, de } \\
\text { natureza quantitativa. }\end{array}$ \\
\hline 9 & LILACS & $\begin{array}{l}\text { Rev. Bras. Promoção } \\
\text { Saúde. }\end{array}$ & $\begin{array}{l}\text { NÓBREGA JD, } \\
\text { et al. }\end{array}$ & $\begin{array}{l}\text { Atenção à saúde e surdez: } \\
\text { Desafios para implantação } \\
\text { da rede de cuidados à } \\
\text { pessoa com deficiência/ } \\
2017 .\end{array}$ & $\begin{array}{c}\text { Estudo de reflexão } \\
\text { abordagem qualitativa. }\end{array}$ \\
\hline 10 & LILACS & $\begin{array}{c}\text { Revista Saúde e } \\
\text { Pesquisa. }\end{array}$ & $\begin{array}{l}\text { LOPES RM, et } \\
\text { al. }\end{array}$ & $\begin{array}{l}\text { Comunicação do surdo com } \\
\text { profissionais de saúde na } \\
\text { busca da integralidade/ } \\
2017 .\end{array}$ & Estudo qualitativo. \\
\hline
\end{tabular}

Fonte: Vieira KA, et al., 2021.

\section{DISCUSSÃO}

\section{Déficit no atendimento prestado ao paciente surdo}

Na concepção de gerar uma assistência integral e qualificada à saúde e de viabilizar mudanças sociais e estruturais, torna-se indispensável que o cidadão seja reconhecido por suas particularidades, valorizando seus direitos de igualdade e sua identidade cultural. Sendo assim, é preciso entender os fatores que os afastam das unidades de saúde. As dificuldades comunicacionais atuam diretamente na visão que os pacientes surdos possuem a respeito do atendimento oferecido, além de intensificar a dependência desses usuários. Os impasses na comunicação e recorrentemente a privação das informações resultam em uma sensação de preconceito e discriminação ligada à deficiência (SANTOS AS e PORTES AJF, 2019).

Cavagna VM, et al. (2017) afirma que existem diversas barreiras nos serviços prestados aos pacientes com surdez, e as que mais se destacam são o impasse linguístico e a ausência de confiança do cliente no profissional ouvinte, pois, muitas vezes, o surdo é comparado a deficientes mentais. $O$ fato de ainda existir 
embates na comunicação, pode ser explicado pela predisposição negativa da população em fazer julgamentos depreciativos sobre estes sujeitos. Visto que, muitas pessoas possuem dificuldades em notar os indivíduos com deficiência como seres humanos produtivos e por acreditarem que eles são inferiores. Por consequência, muitos se sentem discriminados, destratados e indignados com a assistência promovida, resultando no afastamento desses indivíduos nas instituições de saúde e no demérito no relacionamento com a equipe.

A lacuna no acolhimento da relação profissional-cliente produz sentimentos negativos, tal qual angústia, medo, insegurança, impaciência, gerando o distanciamento do servidor com estes pacientes. A compreensão pelo surdo é realizada a partir de uma íntegra comunicabilidade, entretanto, logo após as consultas em saúde essa população ainda mantém a ausência do entendimento sobre seus diagnósticos e suas formas de tratamento, essas circunstâncias solidificam os bloqueios comunicacionais entre esse público e os trabalhadores de saúde (SANTOS AS e PORTES AJF, 2019).

Santos AS e Portes AJF (2019) explica em seus estudos que $82 \%$ dos sujeitos com surdez não compreendem seu diagnóstico a partir das estratégias de comunicação usadas pelo profissional de saúde, e $70 \%$ não conseguem assimilar as orientações sobre seu tratamento, como também $83 \%$ dos surdos em sua pesquisa negaram-se ter obtido um suporte na atenção básica por empregados que dominassem a LIBRAS. Por consequência, $61 \%$ dos participantes do estudo responderam que os funcionários não os entendem caso estiverem sozinhos, além de que, a maneira proposta pelos servidores em saúde para a promoção da comunicabilidade propicia a dificuldade neste processo comunicacional, pois é realizada pela escrita da língua portuguesa, apontando que $70 \%$ utilizam essa técnica e $54 \%$ usam a oralização, sendo estas, classificadas como táticas que mais prejudicam a assistência promovida.

Corroborando com o autor supracitado, Lopes RM, et al. (2017) afirma que a ausência de LIBRAS, uso de mímicas, a utilização da escrita, a aplicação da leitura labial e da oralização durante o atendimento, são estratégias que apresentam falhas pois é necessário compreender que nem todos os surdos fazem o uso da leitura labial ou conseguem oralizar, além disso não são todos que sabem a língua portuguesa, visto que esta é usada como língua acessória e/ou secundária por essa população, como também nem todos são alfabetizados. Sendo assim, nota-se dificuldades no processo de conversação que parte do desinteresse dos profissionais de saúde em melhorar o atendimento, tratando-os como um ouvinte ao invés de se empenhar e com tranquilidade promover a comunicabilidade.

As adversidades na assistência afetam todas as classes do cliente surdo, pois Thomaz MM, et al. (2019) relata em suas análises que o adolescente com surdez também recebe uma precariedade na atenção, no que dispõe da carência de funcionários e ausência da livre demanda de atendimento, estando também atrelada ao preconceito e as indiferenças vivenciadas dentro dos serviços de saúde. De acordo com os participantes, os profissionais os rotulam como adolescentes incapazes, debilitados e adoecidos devido a suas condições auditivas, na qual gera um distanciamento dos clientes surdos e da equipe de saúde. Em suma, nota-se que a comunidade surda não usufrui o serviço do mesmo modo que os ouvintes, devido às barreiras de comunicação, implicando assim na integralidade da assistência, acolhimento e humanização do atendimento e cooperando para a exclusão social.

\section{Desconhecimento sobre LIBRAS}

A receptividade nos sistemas de saúde deve fornecer uma escuta qualificada, tendo em vista, uma resolução positiva ou diminuição dos agravos da situação-problema exposta pelo paciente. A construção de um vínculo é um componente crucial para favorecer o cuidado humanizado e certificar a volta destes indivíduos. Sendo, na área de saúde, a comunicação a melhor forma de promover essa vinculação, já que pode identificar as necessidades que as pessoas apresentam. De tal forma, o despreparo dos colaboradores de saúde acerca de LIBRAS, pode influenciar na distanciação dessa população, posto que, os esclarecimentos sobre suas condições serão repassados para um acompanhante e não propriamente a quem carece da assistência, negligenciando as convicções éticas e desvalorizando a individualidade e autonomia do paciente durante a consulta. O conhecimento básico de palavras em LIBRAS pode proporcionar um desfecho diferenciado no atendimento, no entanto, essa não é uma realidade vivenciada habitualmente no dia-a-dia das pessoas surdas (THOMAZ MM, et al., 2019). 
O enfermeiro é o principal responsável em manter o contato com os pacientes, sua contribuição é baseada na prevenção, no cuidado e na educação. Todavia, essa prática nem sempre é executada de forma eficaz, como mostra Ferreira DRC, et al. (2019) 75\% dos funcionários entrevistados afirmaram que existem entraves na comunicação entre eles e as gestantes surdas, sendo a mais citada a ausência de aprendizado em LIBRAS e a complexidade de assimilação dos sinais e gestos. Diante desse contexto, torna-se ainda mais grave pois, para as gestantes este é um período de grandes mudanças e que exige de uma atenção especial à saúde, em razão do aumento dos números de riscos e possíveis complicações. Em virtude disso, apenas $5 \%$ dos entrevistados relataram se comunicar por LIBRAS durante 0 atendimento prestado, embora $76,6 \%$ concordam que os colaboradores devem ter conhecimento em LIBRAS.

Em estudos de Reis VSL e Santos AM (2019) 60,8\% dos profissionais referiu conhecer sobre LIBRAS, entretanto nenhum sabe se comunicar através dela. Somente 3,3\% tiveram contato com a língua durante a graduação, como também nenhum trabalhador realizou curso complementar ou educação permanente em saúde sobre LIBRAS. Entretanto, 68,5\% dos servidores que atuavam nas Unidades de Saúde da Família (USF) ou Núcleo Ampliado de Saúde da Família (NASF) prestaram assistência a clientes portadores de surdez em algum momento durante sua atuação. Ademais, $61 \%$ dos participantes revelaram possuírem dificuldades para suceder a consulta e $81 \%$ evidenciaram despreparo para prestar uma consulta adequada para esta população.

\section{Ausência de recursos adequados}

Essa problemática surge desde a formação acadêmica, pois a mesma desconsidera o tecido social e cultural da surdez, focando somente no projeto clínico-hegemônico-patológico da deficiência, o qual demonstra a inaptidão desses profissionais provocando assim o desconforto mútuo na assistência à população surda, causando um déficit na compreensão plena das necessidades desses pacientes (NÓBREGA JD, et al., 2017).

A construção de assuntos específicos na graduação sobre essa temática, como: perda da audição; comunicação profissional-paciente; técnicas para melhorar a escuta efetiva procurando compreender a história desses clientes; abordagem das concepções de surdez e da pessoa surda, incluindo suas dimensões fisiológicas, simbólicas e culturais, além de cursos em educação biopsicossocial sobre esses indivíduos tornam-se essenciais nos cursos da área de saúde, dado que, poderia contribuir ao acesso e melhoria dos surdos nos serviços hospitalares (NÓBREGA JD, et al., 2017).

Portanto, nota-se a relevância da inclusão desses aprendizados e da mesma forma a inserção da LIBRAS aos profissionais desde a graduação, visto que, haveria uma criação maior da inter-relação da equipe de saúde com a população surda, estabelecendo uma resolutividade integral perante as questões de saúde desses indivíduos, garantindo a admissão nos ambientes preventivos, curativos e reabilitadores, promoveria a criação de programas de saúde voltadas para esse público, além de favorecer o apoio na capacitação dos servidores do SUS para a linguagem em LIBRAS (FERREIRA DRC, et al., 2019).

Reconhecida perante a Lei, a LIBRAS designa a cultura e identidade dos surdos, sendo assim, sua magnitude deve ser assistida pelos profissionais de saúde e a ausência de seu domínio torna-se uma obstrução para a mutualidade da relação profissional-paciente. A melhoria da assistência aos clientes surdos requer modificações no ambiente físico dos centros de saúde e a capacitação desses funcionários (SANTOS AS e PORTES AJF, 2019).

Confirmando com o autor supramencionado, Soares IP, et al. (2018) destaca que quanto mais pessoas aprendem LIBRAS maior seria a inclusão social e cultural, e sugere que a melhor solução para uma comunicação eficiente entre a equipe de saúde ouvinte e os surdos seriam a qualificação com cursos à distância. Sendo assim, assegurando que os gestores devem compreender essa demanda e dispor de capacitações para todos os servidores.

Depois de qualificados nessa temática haverá mais facilidade na interação, podendo ser minimizadas as barreiras enfrentadas. Entender sobre as estratégias no processo comunicacional com os cidadãos surdos, 
compreendendo suas limitações na prática, auxiliar os funcionários no cuidado para com eles, e igualmente cooperar juntamente com o gestor na organização dos serviços visando as necessidades desses usuários (NÓBREGA JD, et al., 2017).

Esses indivíduos necessitam de utensílios culturais e linguisticamente preparados para a comunidade surda e que só se comunicam por meio da LIBRAS, facilitando assim a assimilação de doenças, uso de fármacos, e principalmente o acesso igualitário nas educações em saúde, ferramentas de revistas, jornais, sites, ou exibições de televisão legendadas. Os surdos apresentam fraco nível de informações, com fonte limitadas e sem disponibilidade sobre educação-saúde, demonstrando desprovimento neste tipo de conhecimento (OLIVEIRA YCA, et al., 2015).

A ausência de recursos adequados e da infraestrutura especializada para o acolhimento desses sujeitos resultam em estratégias improvisadas, que limitam o reconhecimento das suas necessidades, implicando na prescrição segura dos tratamentos, devido à inconsistência na coleta de dados e na formulação do histórico desses pacientes. Os profissionais lamentam a inexistência de artifícios interativos ou recursos audiovisuais que pudessem ajudá-los nas consultas oferecidas, favorecendo a compreensão a respeito das suas queixas e como informá-las sobre (FRANÇA EG, et al., 2016).

Por conseguinte, faz-se necessário atividades entre a gestão dos serviços, instituições de ensino, profissionais da saúde e a comunidade, com o propósito de acolher as necessidades desta população e fornecer os direitos básicos de forma integral, respeitando suas vivências e culturas. Diante disso, os trabalhadores alcançarão desenvolver atendimentos eficientes e incrementar uma atenção integral como fator primordial nas estratégias em saúde (FRANÇA EG, et al., 2016).

\section{CONSIDERAÇÕES FINAIS}

A pessoa surda encontra um cenário com muitos obstáculos e preconceitos entre a execução integral da comunicação destes sujeitos com os profissionais, devido à desqualificação em LIBRAS, carência de recursos e estímulos dos gestores das instituições de saúde. Portanto, cabe aos gestores das unidades de saúde propiciar um ambiente de trabalho inclusos a todos e sem julgamentos precoces, adotando recursos audiovisuais. Assim como, ofertar cursos e campanhas tanto para a comunidade local quanto aos servidores, seguindo os primórdios do SUS. Semelhantemente, é necessário que haja estímulos de aprendizado sobre estes clientes desde a graduação dos enfermeiros e técnicos em enfermagem, com o propósito de aprimorar os atendimentos. Igualmente, é fundamental que ocorra mais pesquisas científicas sobre este tema, demonstrando à sociedade o quão é importante esse amparo aos direitos dos surdos.

\section{REFERÊNCIAS}

1. ANCHIETA EVB. Suicídio e surdez: A saúde mental não acessível. Revista lbero-americana de Humanidades, Ciências e Educação, 2020; 6(6).

2. BRASIL. Constituição da República Federativa do Brasil. 1988.

3. BRASIL. Decreto no 5.626, de 22 de dezembro de 2005.

4. COFEN. Resolução do Conselho Federal de Enfermagem - COFEN № 564/2017.

5. CAVAGNA VM, et al. O paciente surdo e suas vivências no sistema de saúde: uma interface com a enfermagem. Revista Enfermagem atual, 2017.

6. DUARTE SBR, et al. Aspectos históricos e socioculturais da população surda. História, Ciências, Saúde, 2013; 20(4): 1713-1734.

7. FERREIRA, DRC, et al. Assistência à gestante surda: barreiras de comunicação encontradas pela equipe de saúde. Saúde em Redes, 2019; 5(3): 31-42.

8. FRANÇA, EG, et al. Dificuldades de profissionais na atenção à saúde da pessoa com surdez severa. CIENCIA Y ENFERMERIA XXII, 2016; (3): 107-116.

9. FRANCISQUETI V, et al. Sentimentos da equipe de enfermagem ao atender um paciente com deficiência auditiva: desafios do cuidado. Revista Educação Artes e Inclusão, 2017; 13(3).

10. LANNA J, MARTINS MC. História do Movimento Político das Pessoas com Deficiência no Brasil. Brasília: Secretaria de Direitos Humanos. Secretaria Nacional de Promoção dos Direitos da Pessoa com Deficiência, 2010; 443p.

11. LOPES RM, et al. Comunicação do surdo com profissionais de saúde na busca da integralidade. Revista Saúde e Pesquisa, 2017. 
12. NASCIMENTO BD, et al. "Tenho um paciente surdo, e agora?": guia para atendimento e anamnese em acolhimento de enfermagem. Braz. J. Hea. Rev., 2020; 3(4): 10470-10482.

13. NÓBREGA JD, et al. Atenção à saúde e surdez: desafios para implantação da rede de cuidados à pessoas com deficiência. Rev. Bra. Promoç. Saúde, 2017; 30(3): 1-10.

14. OLIVEIRA WSR, et al. Desenvolvimento e aplicação de uma tecnologia educacional para auxiliar a comunicação entre o deficiente auditivo e enfermeiro: relato de experiência. Revista Eletrônica Acervo Saúde, 2020.

15. OLIVEIRA YCA, et al. Comunicação como ferramenta essencial para assistência à saúde dos surdos. Physis Revista de Saúde Coletiva, 2015; 25(1): 307-320.

16. OLIVEIRA YCA, et al. Conhecimento e fonte de informações de pessoas surdas sobre saúde e doença. Interface Comunicação Saúde Educação, 2015; 19(54): 549-60.

17. REISVSL, SANTOS AM. Conhecimento e experiência de profissionais das Equipes de Saúde da Família no atendimento de pessoas surdas. Rev. CEFAC, 2019; 21(1): e5418.

18. SANTOS AS, PORTES AJF. Percepções de sujeitos surdos sobre a comunicação na Atenção Básica à Saúde. Rev. Latino - AM. Enfermagem, 2019; 27: e3127.

19. SOARES IP, et al. Como eu falo com você? A comunicação do enfermeiro com o usuário surdo. Rev. Baiana Enferm., 2018; 32: 25978.

20. SOUZA MFNS, et al. Principais dificuldades e obstáculos enfrentados pela comunidade surda no acesso à saúde: uma revisão integrativa de literatura. Rev. CEFAC, 2017; 19(3): 395-405.

21. THOMAZ MM, et al. Acessibilidade do adolescente com deficiência auditiva aos serviços de saúde. Rev. Eletrônica Enferm., 2019; 21: 55502.

22. TRECOSSI MO, ORTIGARA EPF. Importância e eficácia das consultas de enfermagem ao paciente surdo. Revista de Enfermagem, 2013; 9(9): 60-69. 